\title{
The all-on-four concept may be a viable treatment option for edentulous rehabilitation
}

\author{
Abstracted from \\ Patzelt SB, Bahat O, Reynolds MA, Strub JR. \\ The all-on-four treatment concept: a systematic review. \\ Clin Implant Dent Relat Res 2014; 16: 836-855. doi: 10.1111/cid.12068. \\ Address for correspondence: Dr Sebastian BM Patzelt, Department of Periodontics, School of Dentistry, \\ University of Maryland, 650 West Baltimore Street, Baltimore, MD 21201, USA. E-mail: spatzelt@umaryland.edu
}

\section{Question: In edentulous adult patients, what is the effectiveness of all-on-four treatment concept in terms of implant survival, fixed dental prosthesis (FDP) survival and bone level changes?}

Data sources Medline, the Cochrane Library, Google and handsearches of systematic reviews and bibliographies from related journals in English and German up to August 2012.

Study selection Three reviewers independently searched for clinical trials that assessed the success rates of placing two anterior and two posterior tilted dental implants in humans either in the maxilla or mandible according to the all-on-four treatment concept. Inclusion limited studies with a minimum follow-up period of one year.

Data extraction and synthesis The primary outcome measure was the failure rate of implants. The secondary outcomes were prosthesis failure and marginal bone loss/bone level changes assessed through radiological examination. Failure rate was used to calculate standard deviations (SDs) of implants, whereas the mean success rate was used for dental implants and prostheses. Evaluation and quality assessment of articles and data extraction were performed by three independent reviewers. These reviewers estimated risk of bias by assessing the trial quality using a criteria form focused on the trial design, specification of inclusion/exclusion criteria, performance of surgery, outcome measure provided, radiographic examination of marginal bone level change as well as its evaluation and completeness of follow-up. Outcome measures were based on weighted means using a variance components analysis.

Results Thirteen studies, including 4,804 dental implants (2,000 maxilla and 2,804 mandible) placed in 1,201 jaws met the inclusion criteria. Nine were prospective trials, three retrospective studies and one longitudinal trial. All studies except one were considered to be at high risk of bias. Seventy-four (37 axially, 37 tilted) dental implants failed, with most failures (74\%) within the first 12 months. Fifty-seven out of 1,201 prostheses failed but were repairable. The major prosthetic complication was the fracture of the all-acrylic prostheses. At 36 months the mean cumulative survival rates for implants and prostheses were $99.0 \pm 1.0 \%(S D)$ and $99.9 \pm 0.3 \%(S D)$, respectively with an average bone loss of $1.3 \pm 0.4 \mathrm{~mm}(\mathrm{SD})$. There were no statistically significant differences in the clinical outcomes between maxillary versus mandibular arches and axially versus tilted placed implants.

Conclusions The available evidence shows a promising short-term prognosis for the all-on-four treatment concept. However the evidence is limited by the quality of the available studies and the paucity of clinical trials of greater than five years.

\section{Commentary}

In the edentulous jaw, severe atrophy of the alveolar ridge increases in severity over time. Implant-retained or -supported prostheses provide a higher degree of patient satisfaction than traditional removable denture. ${ }^{1,2,3}$ The all-on-four protocol consists of four implants in the anterior part of an edentulous arch to support an immediately-loaded provisional prosthesis and to avoid grafting procedures. ${ }^{4} 5$ This design may provide an efficient option to oral rehabilitation of the edentulous jaw. $4,6,7$

The review question, inclusion criteria and search strategy were clearly described. However, all were not adequately addressed. However, authors compared outcome measures restricted to a follow-up period of 36 months based on weighted means using a variance components analysis. The review is limited to English and German language articles. The correct clinical studies designs (ie cohort and clinical trials) were included, with the vast majority of them being prospective. There was no assessment of selection agreement between the three independent reviewers (missing Kappa value). The quality assessment questionnaire used to evaluate the studies is not valid.

The overall review findings suggest that the individual studies show promise of the all-on-four approach as an option for the oral rehabilitation of the edentulous patient. However, the authors acknowledge the limitations of the results by mentioning that the low failure rate $(1.5 \%)$ must be considered cautiously given the relatively short follow-up periods ( $\leq 36$ months) and high potential risks of bias. The results of this review are consistent with the recently published (ie, 2012) conclusions of one meta-analysis and two systematic reviews that ask a similar clinical question. ${ }^{4,6,7}$ This further supports the use of posterior tilted implants as an alternative to placing axial implants in bone grafted sites. Generalisability may be a concern since only two proprietary dental implants (BIOMET $3 \mathrm{i}$ and Nobel Biocare) were used in the included studies, and most studies were performed by clinicians who are all-on-four experts.

Current evidence suggests that the all-on-four treatment concept may be a promising approach for the oral rehabilitation of the edentulous jaw. This option potentially offers the patient an efficient treatment outcome with a higher patient quality of life as compared to the conventional extended dental implant treatment approach or conventional removable prostheses. However, the lack 
of good quality long-term studies makes the all-on-four concept still experimental. As such, clinicians and patients must consider this caveat in their clinical decision-making process.

Kelvin I Afrashtehfar McGill University Faculty of Dentistry, Montreal, Canada

1. Att W, Stappert C. Implant therapy to improve quality of life. Quintessence Int 2003 34: 573-581.

2. Engquist B, Bergendal T, Kallus T, Linden U. A retrospective multicenter evaluation of osseointegrated implants supporting overdentures. Int J Oral Maxillofac Implants 1988; 3: 129-134.
3. The McGill consensus statement on overdentures. Quintessence Int 2003; 34: 78-79.

4. Del Fabbro M, Bellini CM, Romeo D, Francetti L. Tilted implants for the rehabilitation of edentulous jaws: a systematic review. Clin Implant Dent Relat Res 2012; 14: 612-621.

5. Heydecke G, Zwahlen M, Nicol A, et al. What is the optimal number of implants for fixed reconstructions: a systematic review. Clin Oral Implants Res 2012; 23: 217-228.

6. Ata-Ali J, Penarrocha-Oltra D, Candel-Marti E, Penarrocha-Diago M. Oral rehabilitation with tilted dental implants: a metaanalysis. Med Oral Patol Oral Cir Bucal 2012; 17: e582-e587.

7. Menini M, Signori A, Tealdo T, et al. Tilted implants in the immediate loading rehabilitation of the maxilla: a systematic review. J Dent Res 2012; 91: 821-827.

Evidence-Based Dentistry (2016) 17, 56-57. doi:10.1038/sj.ebd.6401173 